\title{
PRECISE ASYMPTOTICS OF MOVING AVERAGE PROCESS UNDER $\phi$-MIXING ASSUMPTION
}

\author{
JIE LI
}

\begin{abstract}
In the paper by Liu and Lin (Statist. Probab. Lett. 76 (2006), no. 16, 1787-1799), a new kind of precise asymptotics in the law of large numbers for the sequence of i.i.d. random variables, which includes complete convergence as a special case, was studied. This paper is devoted to the study of this new kind of precise asymptotics in the law of large numbers for moving average process under $\phi$-mixing assumption and some results of Liu and Lin [6] are extended to such moving average process.
\end{abstract}

\section{Introduction}

Let $\left\{\xi_{i},-\infty<i<\infty\right\}$ be a doubly infinite sequence of identically distributed random variables with zero means and finite variances, and let $\left\{a_{i},-\infty<i<\right.$ $\infty\}$ be an absolutely summable sequence of real numbers. Let

$$
X_{k}=\sum_{i=-\infty}^{+\infty} a_{i+k} \xi_{i}, \quad k \geq 1,
$$

be a moving average process based on $\left\{\xi_{i},-\infty<i<\infty\right\}$. Denote $S_{n}=$ $\sum_{k=1}^{n} X_{k}(n \geq 1)$ as the sequence of partial sums.

Under the assumption that $\left\{\xi_{i},-\infty<i<\infty\right\}$ is a sequence of independent identically distributed random variables, many limiting results have been obtained for the moving average process $\left\{X_{k}, k \geq 1\right\}$. Burton and Dehling [2] got a large deviation principle; Yang [8] established the central limit theorem (CLT) and the law of the iterated logarithm (LIL); Li et al. [4] obtained the complete convergence. Under the assumption of dependence, Zhang [9] generalized the results of Li's to $\phi$-mixing with slowly varying functions; Li [5] established the theorem of precise asymptotics in the law of large numbers of moving average process under $\phi$-mixing as follows:

Received September 25, 2010; Revised March 31, 2011.

2010 Mathematics Subject Classification. Primary 60F05, 60G10.

Key words and phrases. complete moment convergence, moving average, $\phi$-mixing, precise asymptotics.

Research supported by foundation of Zhejiang Educational Committee (No. Y201120141). 
Theorem A. Suppose that $\left\{X_{k}, k \geq 1\right\}$ is defined as in (1.1), where $\left\{a_{i},-\infty<\right.$ $i<\infty\}$ is a sequence of real number with $\sum_{i=-\infty}^{\infty}\left|a_{i}\right|<\infty$, and $\left\{\xi_{i},-\infty<\right.$ $i<\infty\}$ is a sequence of identically distributed $\phi$-mixing random variables with $\mathrm{E} \xi_{1}=0, \quad 0<\mathrm{E} \xi_{1}^{2}<\infty, 0<\sigma^{2}=\mathrm{E} \xi_{1}^{2}+2 \sum_{i=2}^{n} \mathrm{E} \xi_{1} \xi_{i}<\infty$, and $\sum_{m=1}^{\infty} \phi^{1 / 2}(m)<\infty$. For $1 \leq p<2$ and $r>p$, if $\mathrm{E}\left|\xi_{1}\right|^{r}<\infty$, then

$$
\lim _{\epsilon \searrow 0} \epsilon^{2(r-p) /(2-p)} \sum_{n=1}^{\infty} n^{r / p-2} \mathrm{P}\left\{\left|S_{n}\right| \geq n^{1 / p} \epsilon\right\}=\frac{p}{r-p} \mathrm{E}|Z|^{2(r-p) /(2-p)},
$$

where $Z \sim N\left(0, \sigma^{2}\left(\sum_{i=-\infty}^{\infty} a_{n i}\right)^{2}\right)$.

Substituting $p=1, r=2$ into (1.2), we obtain

$$
\lim _{\epsilon \searrow 0} \epsilon^{2} \sum_{n=1}^{\infty} \mathrm{P}\left\{\left|S_{n}\right| \geq n \epsilon\right\}=\mathrm{E}|Z|^{2} .
$$

Here we will discuss a new kind of complete convergence. Liu and Lin [6] have discussed the precise rates of convergence of $\sum_{n=1}^{\infty} n^{-q} \mathrm{E}\left|S_{n}\right|^{q} I\left\{\left|S_{n}\right| \geq n \epsilon\right\}$ for sequence of independent identically distributed random variables in the situation of $0 \leq q \leq 2$. Inspired by Liu et al. [6] and Zhang [9], here we consider precise asymptotics in the law of large numbers for moving average process under $\phi$-mixing assumption. It's easy to see that $S_{n}=\sum_{k=1}^{n} X_{k}=\sum_{k=1}^{n} \xi_{k}$ by defining $a_{i+k}=1$ if $i=k$, and $a_{i+k}=0$ otherwise, then $X_{k}$ is a special case of a moving average process. Results analogous to Theorems 1, 2 in Liu and Lin [6] can also be derived for mixing random variables. We mainly use the technique due to Zhang [9] to prove our results based on Theorem A.

Now we give some definitions of mixing random variables. For $m \geq 1$, define

$$
\begin{aligned}
& \phi(m):=\sup _{k \geq 1}\left\{|\mathrm{P}(B \mid A)-\mathrm{P}(B)|, A \in \mathcal{F}_{-\infty}^{k}, \mathrm{P}(A) \neq 0, B \in \mathcal{F}_{k+m}^{\infty}\right\} ; \\
& \rho(m):=\sup _{k \geq 1}\left\{\operatorname{Corr}(U, V) ; U \in \mathrm{E}^{2}\left(\mathcal{F}_{1}^{k}\right), V \in \mathrm{E}^{2}\left(\mathcal{F}_{k+m}^{\infty}\right)\right\},
\end{aligned}
$$

where $\mathcal{F}_{a}^{b}=\sigma\left(\xi_{i}, a \leq i \leq b\right) . \quad\left\{\xi_{i}, i \geq 1\right\}$, a sequence of random variables, is called $\phi$-mixing if $\phi(m) \rightarrow 0$ and $\rho$-mixing if $\rho(m) \rightarrow 0$ as $m \rightarrow \infty$. It is well known that $\rho(m) \leq 2 \phi^{1 / 2}(m)$ and hence a $\phi$-mixing sequence is also $\rho$-mixing.

Throughout the following, $C$ will be used to represent a positive constant although its value may change from one appearance to the next, and $[x]$ to denote the largest integer not greater than $x$.

\section{Main results and some lemmas}

Theorem 2.1. Suppose that $\left\{X_{k}, k \geq 1\right\}$ is defined as in (1.1), where $\left\{a_{i}\right.$, $-\infty<i<\infty\}$ is a sequence of real number with $\sum_{i=-\infty}^{\infty}\left|a_{i}\right|<\infty$, and $\left\{\xi_{i},-\infty<i<\infty\right\}$ is a sequence of identically distributed $\phi$-mixing random variables with $\sum_{m=1}^{\infty} \phi^{1 / 2}(m)<\infty$, and

$$
\mathrm{E} \xi_{1}=0, \quad 0<\mathrm{E} \xi_{1}^{2}<\infty
$$




$$
0<\sigma^{2}=\mathrm{E} \xi_{1}^{2}+2 \sum_{i=2}^{n} \mathrm{E} \xi_{1} \xi_{i}<\infty, \mathrm{E} \xi_{1}^{2} \log ^{+}\left|\xi_{1}\right|<\infty .
$$

Then we have

$$
\lim _{\epsilon \searrow 0} \frac{1}{-\log \epsilon} \sum_{n=1}^{\infty} \frac{1}{n^{2}} \mathrm{E} S_{n}^{2} I\left\{\left|S_{n}\right| \geq n \epsilon\right\}=2 \sigma^{2}\left(\sum_{i=-\infty}^{\infty} a_{n i}\right)^{2}=: 2 \tau^{2} .
$$

Theorem 2.2. Suppose that $\left\{X_{k}, k \geq 1\right\}$ is defined as in (1.1), where $\left\{a_{i}\right.$, $-\infty<i<\infty\}$ is a sequence of real number with $\sum_{i=-\infty}^{\infty}\left|a_{i}\right|<\infty$, and $\left\{\xi_{i},-\infty<i<\infty\right\}$ is a sequence of identically distributed $\phi$-mixing random variables with $\sum_{m=1}^{\infty} \phi^{1 / 2}(m)<\infty$, and

$$
\mathrm{E} \xi_{1}=0, \quad 0<\mathrm{E} \xi_{1}^{2}<\infty, \quad 0<\sigma^{2}=\mathrm{E} \xi_{1}^{2}+2 \sum_{i=2}^{n} \mathrm{E} \xi_{1} \xi_{i}<\infty .
$$

Then we have

$$
\lim _{\epsilon \searrow 0} \epsilon^{2-p} \sum_{n=1}^{\infty} \frac{1}{n^{p}} \mathrm{E}\left|S_{n}\right|^{p} I\left\{\left|S_{n}\right| \geq n \epsilon\right\}=\frac{2}{2-p} \tau^{2}
$$

for $0<p<2$.

It needs the following lemmas to prove the above results.

Lemma 2.1 ([2]). Let $\sum_{i=-\infty}^{+\infty} a_{i}$ be an absolutely convergent series of real numbers with $a=\sum_{i=-\infty}^{+\infty} a_{i}$ and $k \geq 1$. Then

$$
\lim _{n \rightarrow \infty} \frac{1}{n} \sum_{i=-\infty}^{+\infty}\left|\sum_{j=i+1}^{i+n} a_{j}\right|^{k}=|a|^{k} .
$$

Lemma $2.2([7])$. Let $\left\{X_{i}, i \geq 1\right\}$ be a sequence of $\phi$-mixing random variables with zero means and finite second moments. Let $S_{n}=\sum_{i=1}^{n} X_{i}$. If exists $C_{n}$ such that $\max _{1 \leq i \leq n} \mathrm{E} S_{n}^{2} \leq C_{n}$, then for all $q \geq 2$, there exists $C=C(q, \phi(\cdot))$ such that

$$
\mathrm{E} \max _{1 \leq i \leq n}\left|S_{i}\right|^{q} \leq C\left(C_{n}^{q / 2}+\mathrm{E} \max _{1<i \leq n}\left|X_{i}\right|^{q}\right) .
$$

\section{Proof of Theorem 2.1}

Without loss of generality, we assume $\tau^{2}=1$. It's easy to see

$$
\sum_{n=1}^{\infty} \frac{1}{n^{2}} \mathrm{E} S_{n}^{2} I\left\{\left|S_{n}\right| \geq n \epsilon\right\}=\epsilon^{2} \sum_{n=1}^{\infty} \mathrm{P}\left(\left|S_{n}\right| \geq n \epsilon\right)+\sum_{n=1}^{\infty} \frac{1}{n^{2}} \int_{n \epsilon}^{\infty} 2 x \mathrm{P}\left(\left|S_{n}\right| \geq x\right) d x .
$$

Proof of Theorem 2.1. Let $b(\epsilon)=\left[\epsilon^{-2}\right]$. With (1.3), we only need to prove

$$
\lim _{\epsilon \searrow 0} \frac{1}{-\log \epsilon} \sum_{n=1}^{\infty} \frac{1}{n^{2}} \int_{n \epsilon}^{\infty} 2 x \mathrm{P}\left(\left|S_{n}\right| \geq x\right) d x=2 .
$$


By Proposition 3.1 in [6], (3.2) holds if we can prove the following propositions.

Proposition 3.1. One has

$$
\lim _{\epsilon \searrow 0} \frac{1}{-\log \epsilon} \sum_{n=1}^{b(\epsilon)} \frac{1}{n^{2}}\left|\int_{n \epsilon}^{\infty} 2 x P\left(\left|S_{n}\right| \geq x\right) d x-\int_{n \epsilon}^{\infty} 2 x P(|N| \geq x / \sqrt{n}) d x\right|=0 .
$$

Proof. Obviously,

$$
\begin{aligned}
& \sum_{n=1}^{b(\epsilon)} \frac{1}{n^{2}}\left|\int_{n \epsilon}^{\infty} 2 x \mathrm{P}\left(\left|S_{n}\right| \geq x\right) d x-\int_{n \epsilon}^{\infty} 2 x \mathrm{P}(|N| \geq x / \sqrt{n}) d x\right| \\
= & \sum_{n=1}^{b(\epsilon)}\left|\int_{0}^{\infty} 2(x+\epsilon) \mathrm{P}\left(\left|S_{n}\right| \geq n(x+\epsilon)\right) d x-\int_{0}^{\infty} 2(x+\epsilon) \mathrm{P}(|N| \geq \sqrt{n}(x+\epsilon)) d x\right| \\
\leq & \sum_{n=1}^{b(\epsilon)} \frac{1}{n} \int_{0}^{\infty} 2 n(x+\epsilon)\left|\mathrm{P}\left(\left|S_{n}\right| \geq n(x+\epsilon)\right)-\mathrm{P}(|N| \geq \sqrt{n}(x+\epsilon))\right| d x \\
\leq & \sum_{n=1}^{b(\epsilon)} \frac{1}{n}\left(\Delta_{n 1}+\Delta_{n 2}+\Delta_{n 3}\right),
\end{aligned}
$$

where

$$
\begin{aligned}
& \Delta_{n 1}=\int_{0}^{1 / \sqrt{n} \Delta_{n}{ }^{1 / 4}} 2 n(x+\epsilon)\left|\mathrm{P}\left(\left|S_{n}\right| \geq n(x+\epsilon)\right)-\mathrm{P}(|N| \geq \sqrt{n}(x+\epsilon))\right| d x, \\
& \Delta_{n 2}=\int_{1 / \sqrt{n} \Delta_{n}{ }^{1 / 4}}^{\infty} 2 n(x+\epsilon) \mathrm{P}\left(\left|S_{n}\right| \geq n(x+\epsilon)\right) d x \\
& \Delta_{n 3}=\int_{1 / \sqrt{n} \Delta_{n}{ }^{1 / 4}}^{\infty} 2 n(x+\epsilon) \mathrm{P}(|N| \geq \sqrt{n}(x+\epsilon)) d x \\
& \Delta_{n}=\sup _{x}\left|\mathrm{P}\left(\left|S_{n}\right| \geq \sqrt{n} x\right)-\mathrm{P}(|N| \geq x)\right| .
\end{aligned}
$$

By the Berry-Esseen theorem for a moving average process generated by $\phi$ mixing sequence, we get $\Delta_{n} \rightarrow 0$ as $n \rightarrow \infty$.

Since $n \leq b(\epsilon)$ implies $\sqrt{n} \epsilon \leq 1$, we can get

$$
\begin{aligned}
\Delta_{n 1} & \leq \int_{0}^{1 / \sqrt{n} \Delta_{n}{ }^{1 / 4}} 2 n(x+\epsilon) \Delta_{n} d x \\
& \leq n \Delta_{n}\left(\frac{1}{\sqrt{n} \Delta_{n}{ }^{1 / 4}}+\epsilon\right)^{2} \leq\left(\Delta_{n}^{1 / 4}+\Delta_{n}^{1 / 2}\right)^{2} .
\end{aligned}
$$

For $\Delta_{n 3}$, by the Markov inequality, we have

$$
\Delta_{n 3} \leq C n \int_{1 / \sqrt{n} \Delta_{n}^{1 / 4}}^{\infty} \frac{1}{n^{2}(x+\epsilon)^{3}} d x \leq C \Delta_{n}^{1 / 2} .
$$


By (3.4) and (3.5), we obtain

$$
\lim _{\epsilon \searrow 0} \frac{1}{-\log \epsilon} \sum_{n=1}^{b(\epsilon)}\left\{\frac{1}{n}\left(\Delta_{n 1}+\Delta_{n 3}\right)\right\}=0 .
$$

Let

$$
\begin{aligned}
& \xi_{i}^{\prime}=\xi_{i} I\left\{\left|\xi_{i}\right| \leq \lambda\right\}, \quad \xi_{i}^{\prime \prime}=\xi_{i} I\left\{\left|\xi_{i}\right|>\lambda\right\} \\
& S_{n}^{\prime}=\sum_{k=1}^{n} X_{k}^{\prime}=\sum_{i=-\infty}^{+\infty} a_{n i} \xi_{i}^{\prime}, \quad S_{n}^{\prime \prime}=\sum_{i=-\infty}^{+\infty} a_{n i} \xi_{i}^{\prime \prime} .
\end{aligned}
$$

Therefore,

$$
\begin{aligned}
\sum_{n=1}^{b(\epsilon)} \frac{1}{n} \Delta_{n 2} \leq & \sum_{n=1}^{b(\epsilon)} \int_{1 / \sqrt{n} \Delta_{n}{ }^{1 / 4}}^{\infty} 2(x+\epsilon) \mathrm{P}\left(\left|S_{n}^{\prime}\right| \geq \frac{n(x+\epsilon)}{2}\right) d x \\
& +\sum_{n=1}^{b(\epsilon)} \int_{1 / \sqrt{n} \Delta_{n}{ }^{1 / 4}}^{\infty} 2(x+\epsilon) \mathrm{P}\left(\left|S_{n}^{\prime \prime}\right| \geq \frac{n(x+\epsilon)}{2}\right) d x \\
= & : I_{1}+I_{2},
\end{aligned}
$$

and

$$
I_{1} \leq C \sum_{n=1}^{b(\epsilon)} \int_{0}^{\infty} \frac{\mathrm{E}\left|X_{1}^{\prime}\right|^{4}}{n^{2}(x+\epsilon)^{3}} d x \leq C \mathrm{E}\left|X_{1}^{\prime}\right|^{4} \sum_{n=1}^{b(\epsilon)} \frac{1}{n^{2} \epsilon} \leq C \mathrm{E}\left|\xi_{1}^{\prime}\right|^{4} .
$$

Thus, we have

$$
\lim _{\epsilon \searrow 0} \frac{1}{-\log \epsilon} I_{1}=0 .
$$

Observe that $\sum_{k=1}^{n} X_{k}=\sum_{i=-\infty}^{\infty} \sum_{k=1}^{n} a_{k+i} \xi_{i}=\sum_{i=-\infty}^{\infty} a_{n i} \xi_{i}$, where $a_{n i}=$ $\sum_{k=1}^{n} a_{k+i}$. By Lemma 2.1, we can assume

$$
\sum_{i=-\infty}^{\infty}\left|a_{n i}\right|^{t} \leq n, \quad t \geq 1 \text { and } \tilde{a}=\sum_{i=-\infty}^{\infty}\left|a_{i}\right| \leq 1 .
$$

Let $T_{n}^{\prime \prime}=\sum_{i=-\infty}^{\infty} a_{n i} \xi_{i}^{\prime \prime} I\left\{\left|a_{n i} \xi_{i}^{\prime \prime}\right| \leq n(x+\epsilon)\right\}$. Then

$$
\begin{aligned}
\left|\mathrm{E} T_{n}^{\prime \prime}\right| & =\left|\sum_{i=-\infty}^{\infty} a_{n i} \mathrm{E} \xi_{1}^{\prime \prime} I\left\{\left|a_{n i} \xi_{1}^{\prime \prime}\right|>n(x+\epsilon)\right\}\right| \\
& \leq \sum_{i=-\infty}^{\infty}\left|a_{n i}\right| \mathrm{E}\left|\xi_{1}^{\prime \prime}\right| I\left\{\tilde{a}\left|\xi_{1}^{\prime \prime}\right|>n(x+\epsilon)\right\} \\
& \leq n \mathrm{E}\left|\xi_{1}^{\prime \prime}\right| I\left\{\left|\xi_{1}^{\prime \prime}\right|>n(x+\epsilon)\right\} \\
& \leq \frac{\mathrm{E}\left|\xi_{1}^{\prime \prime}\right|^{2} I\left\{\left|\xi_{1}^{\prime \prime}\right|>n(x+\epsilon)\right\}}{x+\epsilon}
\end{aligned}
$$


In the situation of $x \in\left(1 / \sqrt{n} \Delta_{n}^{1 / 4}, \infty\right)$, we have

$$
\frac{1}{n(x+\epsilon)}\left|\mathrm{E} T_{n}^{\prime \prime}\right| \leq \frac{1}{n(x+\epsilon)^{2}} \mathrm{E}\left|\xi_{1}^{\prime \prime}\right|^{2} I\left\{\left|\xi_{1}^{\prime \prime}\right|>n(x+\epsilon)\right\}<\frac{\mathrm{E}\left|\xi_{1}^{\prime \prime}\right|^{2}}{\Delta_{n}^{1 / 2}}<\epsilon .
$$

Thus,

$$
\begin{gathered}
I_{2} \leq C \sum_{n=1}^{b(\epsilon)} \int_{1 / \sqrt{n} \Delta_{n}^{1 / 4}}^{\infty}(x+\epsilon)\left[\mathrm{P}\left(\sup _{i}\left|a_{n i} \xi_{i}^{\prime \prime}\right|>n(x+\epsilon)\right)\right. \\
\left.\quad+\mathrm{P}\left(\left|T_{n}^{\prime \prime}-E T_{n}^{\prime \prime}\right| \geq n(x+\epsilon) / 4\right)\right] d x \\
=: I_{21}+I_{22} .
\end{gathered}
$$

Set $I_{n j}=\left\{j \in \mathcal{L}, 1 /(j+1)<\left|a_{n j}\right| \leq 1 / j, j=1,2, \ldots\right\}$, then $\bigcup_{j \geq 1} I_{n j}=\mathcal{L}$ (see [4]). We can get

$$
\sum_{j=1}^{k} \sharp I_{n j} \leq n(k+1) .
$$

Therefore,

$$
\begin{aligned}
& \mathrm{P}\left\{\sup _{i}\left|a_{n i} \xi_{i}^{\prime \prime}\right|>n(x+\epsilon)\right\} \\
\leq & \sum_{i=-\infty}^{\infty} \mathrm{P}\left\{\left|a_{n i} \xi_{i}^{\prime \prime}\right|>n(x+\epsilon)\right\} \\
\leq & \sum_{j=1}^{\infty} \sum_{i \in I_{n j}} \mathrm{P}\left\{\left|\xi_{1}^{\prime \prime}\right| \geq n j(x+\epsilon)\right\} \\
\leq & \sum_{j=1}^{\infty}\left(\sharp I_{n j}\right) \mathrm{P}\left\{\left|\xi_{1}^{\prime \prime}\right| \geq n j(x+\epsilon)\right\} \\
\leq & \sum_{j=1}^{\infty} \sum_{k \geq j}\left(\sharp I_{n j}\right) \mathrm{P}\left\{n k(x+\epsilon) \leq\left|\xi_{1}^{\prime \prime}\right|<n(k+1)(x+\epsilon)\right\} \\
\leq & \sum_{k=1}^{\infty} \sum_{j=1}^{k}\left(\sharp I_{n j}\right) \mathrm{P}\left\{n k(x+\epsilon) \leq\left|\xi_{1}^{\prime \prime}\right|<n(k+1)(x+\epsilon)\right\} \\
\leq & \sum_{k=1}^{\infty} n(k+1) \mathrm{P}\left\{n k(x+\epsilon) \leq\left|\xi_{1}^{\prime \prime}\right|<n(k+1)(x+\epsilon)\right\} \\
\leq & \frac{\mathrm{E}\left|\xi_{1}^{\prime \prime}\right| I\left\{\left|\xi_{1}^{\prime \prime}\right| \geq n(x+\epsilon)\right\}}{x+\epsilon} .
\end{aligned}
$$

Then

$$
I_{21} \leq C \mathrm{E}\left|\xi_{1}^{\prime \prime}\right| \int_{1 / \sqrt{n} \Delta_{n}^{1 / 4}}^{\infty} \sum_{n=1}^{b(\epsilon)} \sum_{k=n}^{\infty} I\left\{k(x+\epsilon) \leq\left|\xi_{1}^{\prime \prime}\right|<(k+1)(x+\epsilon)\right\} d x
$$




$$
\begin{aligned}
& \leq C \mathrm{E}\left|\xi_{1}^{\prime \prime}\right| \int_{0}^{\infty} \sum_{k=1}^{\infty} k I\left\{k(x+\epsilon) \leq\left|\xi_{1}^{\prime \prime}\right|<(k+1)(x+\epsilon)\right\} d x \\
& \leq C \mathrm{E}\left|\xi_{1}^{\prime \prime}\right|^{2} \int_{0}^{\infty}(x+\epsilon)^{-1} I\left\{\left|\xi_{1}^{\prime \prime}\right| \geq(x+\epsilon)\right\} d x \\
& \leq C \mathrm{E}\left|\xi_{1}^{\prime \prime}\right|^{2} \log ^{+}\left|\xi_{1}^{\prime \prime}\right|-C \mathrm{E}\left|\xi_{1}^{\prime \prime}\right|^{2}(\log \epsilon) .
\end{aligned}
$$

Hence

$$
\lim _{\lambda \rightarrow \infty} \limsup _{\epsilon \searrow 0} \frac{1}{-\log \epsilon} I_{21}=0 .
$$

As for $I_{22}$, note that $\sum_{m=1}^{\infty} \phi^{1 / 2}(m)<\infty$, then

$$
\begin{gathered}
\sup _{-\infty<l \leq m<\infty} \mathrm{E}\left[\sum_{i=l}^{m} a_{n i} \xi_{i}^{\prime \prime} I\left\{\left|a_{n i} \xi_{i}^{\prime \prime}\right| \leq n(x+\epsilon)\right\}\right. \\
\left.-\mathrm{E}\left(\sum_{i=l}^{m} a_{n i} \xi_{i}^{\prime \prime} I\left\{\left|a_{n i} \xi_{i}^{\prime \prime}\right| \leq n(x+\epsilon)\right\}\right)\right]^{2} \\
\leq C \sum_{i=-\infty}^{\infty} \mathrm{E}\left(a_{n i} \xi_{1}^{\prime \prime}\right)^{2} I\left\{\left|a_{n i} \xi_{1}^{\prime \prime}\right| \leq n(x+\epsilon)\right\} .
\end{gathered}
$$

By Lemma 2.2, for $q>2$, we have

$$
\begin{aligned}
I_{22} \leq & \sum_{n=1}^{b(\epsilon)} \int_{1 / \sqrt{n} \Delta_{n}^{1 / 4}}^{\infty} n^{-q}(x+\epsilon)^{1-q}\left\{\left(\sum_{i=-\infty}^{\infty} a_{n i}^{2} \mathrm{E}\left|\xi_{1}^{\prime \prime}\right|^{2} I\left\{\left|a_{n i} \xi_{1}^{\prime \prime}\right| \leq n(x+\epsilon)\right\}\right)^{q / 2}\right. \\
& +\sum_{i=-\infty}^{\infty} \mathrm{E}\left|a_{n i} \xi_{1}^{\prime \prime}\right|^{q} I\left\{\left|a_{n i} \xi_{1}^{\prime \prime}\right| \leq n \epsilon\right\} \\
& \left.+\sum_{i=-\infty}^{\infty} \mathrm{E}\left|a_{n i} \xi_{1}^{\prime \prime}\right|^{q} I\left\{n \epsilon<\left|a_{n i} \xi_{1}^{\prime \prime}\right| \leq n(x+\epsilon)\right\}\right\} d x \\
= & I_{221}+I_{222}+I_{223} .
\end{aligned}
$$

For $I_{221}, q>2$, we have

$$
\begin{aligned}
I_{221} & \leq \sum_{n=1}^{b(\epsilon)} \int_{1 / \sqrt{n} \Delta^{1 / 4}}^{\infty} n^{-q}(x+\epsilon)^{1-q}\left(\sum_{i=-\infty}^{\infty} a_{n i}^{2} \mathrm{E}\left|\xi_{1}^{\prime \prime}\right|^{2}\right)^{q / 2} d x \\
& \leq C \sum_{n=1}^{b(\epsilon)} \frac{1}{n} \Delta_{n}^{\frac{q-2}{4}} .
\end{aligned}
$$

First we estimate $I_{222}$,

$$
I_{222} \leq \sum_{n=1}^{b(\epsilon)} \int_{1 / \sqrt{n} \Delta^{1 / 4}}^{\infty} n^{-q}(x+\epsilon)^{1-q} \sum_{j=1}^{\infty} \sum_{i \in I_{n j}}\left|a_{n i}\right|^{q} \mathrm{E}\left|\xi_{1}^{\prime \prime}\right|^{q} I\left\{\left|a_{n i} \xi_{1}^{\prime \prime}\right| \leq n \epsilon\right\} d x
$$




$$
\begin{aligned}
\leq & C \epsilon^{2-q} \sum_{n=1}^{b(\epsilon)} n^{-q} \sum_{j=1}^{\infty}\left(\sharp I_{n j}\right) j^{-q} \sum_{k=0}^{(j+1) n} \mathrm{E}\left|\xi_{1}^{\prime \prime}\right|^{q} I\left\{k \epsilon \leq\left|\xi_{1}^{\prime \prime}\right|<(k+1) \epsilon\right\} d x \\
\leq & C \epsilon^{2-q} \sum_{n=1}^{b(\epsilon)} n^{-q} \sum_{j=1}^{\infty}\left(\sharp I_{n j}\right) j^{-q}\left\{\sum_{k=0}^{2 n} \mathrm{E}\left|\xi_{1}^{\prime \prime}\right|^{q} I\left\{k \epsilon \leq\left|\xi_{1}^{\prime \prime}\right|<(k+1) \epsilon\right\}\right. \\
& \left.+\sum_{k=2 n+1}^{(j+1) n} \mathrm{E}\left|\xi_{1}^{\prime \prime}\right|^{q} I\left\{k \epsilon \leq\left|\xi_{1}^{\prime \prime}\right|<(k+1) \epsilon\right\}\right\} d x \\
= & : I_{2221}+I_{2222} .
\end{aligned}
$$

Note that

$$
\begin{aligned}
\sum_{j=m}^{\infty}\left(\sharp I_{n j}\right)(j+1)^{-q}(m+1)^{q-1} & \leq \sum_{j=1}^{\infty}\left(\sharp I_{n j}\right)(j+1)^{-1} \\
& \leq \sum_{j=1}^{\infty} \sum_{i \in I_{n j}}\left|a_{n i}\right|=\sum_{i=-\infty}^{\infty}\left|a_{n i}\right| \leq n .
\end{aligned}
$$

Then

$$
\sum_{j=m}^{\infty}\left(\sharp I_{n j}\right) j^{-q} \leq C n m^{-(q-1)} .
$$

Now we estimate $I_{2221}$. By (3.13), we get

$$
\begin{aligned}
I_{2221} & \leq C \epsilon^{2-q} \sum_{n=1}^{b(\epsilon)} n^{1-q} \sum_{k=0}^{2 n} \mathrm{E}\left|\xi_{1}^{\prime \prime}\right|^{q} I\left\{k \epsilon \leq\left|\xi_{1}^{\prime \prime}\right|<(k+1) \epsilon\right\} \sum_{n=[k / 2]}^{b(\epsilon)} n^{1-q} \\
& \leq C \epsilon^{2-q} \sum_{k=0}^{b(\epsilon)} \mathrm{E}\left|\xi_{1}^{\prime \prime}\right|^{q} I\left\{k \epsilon \leq\left|\xi_{1}^{\prime \prime}\right|<(k+1) \epsilon\right\} \\
& \leq C \epsilon^{2-q} \sum_{k=0}^{b(\epsilon)} k^{2-q} \mathrm{E}\left|\xi_{1}^{\prime \prime}\right|^{q} I\left\{k \epsilon \leq\left|\xi_{1}^{\prime \prime}\right|<(k+1) \epsilon\right\} \\
& \leq C \sum_{k=0}^{\infty} \mathrm{E}\left|\xi_{1}^{\prime \prime}\right|^{2} I\left\{k \epsilon \leq\left|\xi_{1}^{\prime \prime}\right|<(k+1) \epsilon\right\}
\end{aligned}
$$

and

$$
\begin{aligned}
I_{2222} & \leq \epsilon^{2-q} \sum_{n=1}^{b(\epsilon)} n^{-q} \sum_{k=2 n+1}^{\infty} \sum_{j \geq k / n-1}\left(\sharp I_{n j}\right) j^{-q} \mathrm{E}\left|\xi_{1}^{\prime \prime}\right|^{q} I\left\{k \epsilon \leq\left|\xi_{1}^{\prime \prime}\right|<(k+1) \epsilon\right\} \\
& \leq \epsilon^{2-q} \sum_{n=1}^{b(\epsilon)} n^{-q} \sum_{k=2 n+1}^{\infty} n\left(\frac{k}{n}\right)^{-(q-1)} \mathrm{E}\left|\xi_{1}^{\prime \prime}\right|^{q} I\left\{k \epsilon \leq\left|\xi_{1}^{\prime \prime}\right|<(k+1) \epsilon\right\}
\end{aligned}
$$


(3.15) $\leq C \mathrm{E}\left|\xi_{1}^{\prime \prime}\right|^{2}$.

$$
\begin{aligned}
& \leq \epsilon^{2-q} \sum_{k=2}^{\infty} \mathrm{E}\left|\xi_{1}^{\prime \prime}\right|^{q} I\left\{k \epsilon \leq\left|\xi_{1}^{\prime \prime}\right|<(k+1) \epsilon\right\} \sum_{n=1}^{[k / 2]} k^{1-q} \\
& \leq C \epsilon^{2-q} \sum_{k=2}^{\infty} k^{2-q} \mathrm{E}\left|\xi_{1}^{\prime \prime}\right|^{q} I\left\{k \epsilon \leq\left|\xi_{1}^{\prime \prime}\right|<(k+1) \epsilon\right\}
\end{aligned}
$$

Now we estimate $I_{223}$, by (3.9), we will get

$$
\begin{aligned}
I_{223} \leq & C \sum_{n=1}^{b(\epsilon)} n^{-q} \mathrm{E}\left|\xi_{1}^{\prime \prime}\right|{ }^{q} I\left\{\left|\tilde{a} \xi_{1}^{\prime \prime}\right|>n \epsilon\right\} \int_{0}^{\infty}(x+\epsilon)^{1-q} \sum_{i=-\infty}^{\infty}\left|a_{n i}\right|^{q} I\left\{\left|a_{n i} \xi_{1}^{\prime \prime}\right| \leq n(x+\epsilon)\right\} d x \\
\leq & C \sum_{n=1}^{b(\epsilon)} n^{-q} \mathrm{E}\left|\xi_{1}^{\prime \prime \prime}\right| I^{I}\left\{\left|\tilde{a}_{1}^{\prime \prime}\right|>n \epsilon\right\} \int_{0}^{\infty}(x+\epsilon)^{1-q} \sum_{j=1}^{\infty}\left(\sharp I_{n j}\right) j^{-q} \sum_{k=0}^{(j+1) n} I\left\{k(x+\epsilon) \leq\left|\xi_{1}^{\prime \prime}\right|<(k+1)(x+\epsilon)\right\} d x \\
\leq & \left.C \sum_{n=1}^{b(\epsilon)} n^{1-q} \mathrm{E}\left|\xi_{1}^{\prime \prime}\right|\right|^{q} I\left\{\left|\xi_{1}^{\prime \prime}\right|>n \epsilon\right\} \int_{0}^{\infty}(x+\epsilon)^{1-q} \sum_{k=0}^{2 n} I\left\{k(x+\epsilon) \leq\left|\xi_{1}^{\prime \prime}\right|<(k+1)(x+\epsilon)\right\} d x \\
& +C \sum_{n=1}^{b(\epsilon)} \mathrm{E}\left|\xi_{1}^{\prime \prime}\right|^{q} I\left\{\left|\xi_{1}^{\prime \prime}\right|>n \epsilon\right\} \sum_{k=2 n+1}^{\infty} k^{1-q} \int_{0}^{\infty}(x+\epsilon)^{1-q} I\left\{k(x+\epsilon) \leq\left|\xi_{1}^{\prime \prime}\right|<(k+1)(x+\epsilon)\right\} d x \\
\leq & C \sum_{n=1}^{b(\epsilon)} n^{-1} \mathrm{E}\left|\xi_{1}^{\prime \prime}\right|^{2} I\left\{\left|\xi_{1}^{\prime \prime}\right|>n \epsilon\right\}+C \sum_{n=1}^{b(\epsilon)}(2 n+1)^{-1} \mathrm{E}\left|\xi_{1}^{\prime \prime}\right| I\left\{\left|\xi_{1}^{\prime \prime}\right|>n(x+\epsilon)\right\} d x \\
(3.16) \leq & C \mathrm{E}\left|\xi_{1}^{\prime \prime}\right|^{2}\left(\log ^{+}\left|\xi_{1}^{\prime \prime}\right|-\log \epsilon\right) .
\end{aligned}
$$

From (3.12) to (3.16), we have

$$
\lim _{\lambda \rightarrow \infty} \lim _{\epsilon} \frac{1}{-\log \epsilon} I_{22}=0 .
$$

Combining (3.6), (3.8), (3.11), and (3.17), (3.3) is derived.

Proposition 3.2. One has

$\lim _{\epsilon \searrow 0} \frac{1}{-\log \epsilon} \sum_{n=b(\epsilon)+1}^{\infty} \frac{1}{n^{2}}\left|\int_{n \epsilon}^{\infty} 2 x P\left(\left|S_{n}\right| \geq x\right) d x-\int_{n \epsilon}^{\infty} 2 x P(|N| \geq x / \sqrt{n}) d x\right|=0$.

Proof. Consider the following:

$$
\begin{aligned}
& \sum_{n=b(\epsilon)+1}^{\infty} \frac{1}{n^{2}}\left|\int_{n \epsilon}^{\infty} 2 x \mathrm{P}\left(\left|S_{n}\right| \geq x\right) d x-\int_{n \epsilon}^{\infty} 2 x \mathrm{P}(|N| \geq x / \sqrt{n}) d x\right| \\
\leq & \sum_{n=b(\epsilon)+1}^{\infty} \int_{0}^{\infty} 2(x+\epsilon) \mathrm{P}(|N| \geq \sqrt{n}(x+\epsilon)) d x+\sum_{n=b(\epsilon)+1}^{\infty} \int_{0}^{\infty} 2(x+\epsilon) \mathrm{P}\left(\left|S_{n}\right| \geq n(x+\epsilon)\right) d x \\
= & M_{1}+M_{2} .
\end{aligned}
$$

For $M_{1}$, we have

(3.19) $\quad M_{1} \leq C \sum_{n=b(\epsilon)+1}^{\infty} \int_{0}^{\infty} 2(x+\epsilon) \frac{1}{n^{2}(x+\epsilon)^{4}} d x \leq C \sum_{n=b(\epsilon)+1}^{\infty} \frac{1}{n^{2} \epsilon^{2}} \leq C$. 
Set $U_{n}^{\prime}=\sum_{i=-\infty}^{\infty} a_{n i} \xi_{i} I\left\{\left|a_{n i} \xi_{i}\right| \leq n(x+\epsilon)\right\}$. And

$$
\begin{aligned}
M_{2} \leq & \sum_{n=b(\epsilon)+1}^{\infty} \int_{0}^{\infty} 2(x+\epsilon)\left[\mathrm{P}\left(\sup _{i}\left|a_{n i} \xi_{i}\right|>n(x+\epsilon)\right)\right. \\
& \left.+\mathrm{P}\left(\left|U_{n}^{\prime}-E U_{n}^{\prime}\right| \geq n(x+\epsilon) / 2\right)\right] d x \\
=: & M_{21}+M_{22} .
\end{aligned}
$$

Now we estimate $M_{21}$. Note that from $\mathrm{E} \xi_{1}^{2} \log ^{+}\left|\xi_{1}\right|<\infty$, we have

$$
\begin{aligned}
M_{21} & \leq C \sum_{n=b(\epsilon)+1}^{\infty} \int_{0}^{\infty} \sum_{k=n}^{\infty} \mathrm{E}\left|\xi_{1}\right| I\left\{k(x+\epsilon) \leq\left|\xi_{1}\right|<(k+1)(x+\epsilon)\right\} d x \\
& \leq C \mathrm{E}\left|\xi_{1}\right|^{2} I\left\{\left|\xi_{1}\right|>\epsilon^{-1}\right\} \int_{0}^{\infty} \frac{I\left\{\left|\xi_{1}\right|>(x+\epsilon)\right\}}{x+\epsilon} d x \\
& \leq C \mathrm{E}\left|\xi_{1}\right|^{2} I\left\{\left|\xi_{1}\right|>\epsilon^{-1}\right\}\left(\log ^{+}\left|\xi_{1}\right|-\log \epsilon\right) .
\end{aligned}
$$

Using the same arguments as those employed in the proof of Proposition 3.1, we have

$$
\begin{aligned}
M_{22} \leq & \sum_{n=b(\epsilon)+1}^{\infty} \int_{0}^{\infty} n^{-q}(x+\epsilon)^{1-q}\left\{\left(\sum_{i=-\infty}^{\infty} \mathrm{E}\left(a_{n i} \xi_{1}\right)^{2} I\left\{\left|a_{n i} \xi_{1}\right| \leq n(x+\epsilon)\right\}\right)^{q / 2}\right. \\
& +\sum_{i=-\infty}^{\infty} \mathrm{E}\left|a_{n i} \xi_{1}\right|^{q} I\left\{\left|a_{n i} \xi_{1}\right| \leq n \epsilon\right\} \\
& \left.+\sum_{i=-\infty}^{\infty} \mathrm{E}\left|a_{n i} \xi_{1}\right|^{q} I\left\{n \epsilon<\left|a_{n i} \xi_{1}\right| \leq n(x+\epsilon)\right\}\right\} d x \\
=: & M_{221}+M_{222}+M_{223} .
\end{aligned}
$$

And

$$
\begin{aligned}
M_{221} & \leq \sum_{n=b(\epsilon)+1}^{\infty} \int_{0}^{\infty} n^{-q / 2}(x+\epsilon)^{1-q}\left(\mathrm{E} \xi_{1}^{2}\right)^{q / 2} d x \\
& \leq C\left(\mathrm{E} \xi_{1}^{2}\right)^{q / 2} \sum_{n=b(\epsilon)+1}^{\infty} n^{-q / 2} \epsilon^{2-q} \leq C .
\end{aligned}
$$

Then

$$
\begin{aligned}
M_{222} & \leq \epsilon^{2-q} \sum_{n=b(\epsilon)+1}^{\infty} n^{-q} \sum_{i=-\infty}^{\infty}\left|a_{n i}\right|^{q} \mathrm{E}\left|\xi_{1}\right|^{q} I\left\{\left|a_{n i} \xi_{1}\right| \leq n \epsilon\right\} \\
& \leq \epsilon^{2-q} \sum_{n=b(\epsilon)+1}^{\infty} n^{-q} \sum_{j=1}^{\infty}\left(\sharp I_{n j}\right) j^{-q} \sum_{k=0}^{(j+1) n} \mathrm{E}\left|\xi_{1}\right|^{q} I\left\{k \epsilon \leq\left|\xi_{1}\right|<(k+1) \epsilon\right\}
\end{aligned}
$$




$$
\begin{aligned}
\leq & \epsilon^{2-q} \sum_{n=b(\epsilon)+1}^{\infty} n^{-q} \sum_{j=1}^{\infty}\left(\sharp I_{n j}\right) j^{-q}\left\{\sum_{k=0}^{2 n}+\sum_{k=2 n+1}^{(j+1) n}\right\} \mathrm{E}\left|\xi_{1}\right|^{q} I\left\{k \epsilon \leq\left|\xi_{1}\right|<(k+1) \epsilon\right\} \\
\leq & C \epsilon^{2-q} \sum_{n=b(\epsilon)+1}^{\infty} n^{1-q} \sum_{k=0}^{2 n} \mathrm{E}\left|\xi_{1}\right|^{q} I\left\{k \epsilon \leq\left|\xi_{1}\right|<(k+1) \epsilon\right\} \\
& +C \epsilon^{2-q} \sum_{n=b(\epsilon)+1}^{\infty} n^{-q} \sum_{k=2 n+1}^{\infty} n\left(\frac{k}{n}\right)^{-(q-1)} \mathrm{E}\left|\xi_{1}\right|^{q} I\left\{k \epsilon \leq\left|\xi_{1}\right|<(k+1) \epsilon\right\} \\
\leq & C \epsilon^{2-q} \sum_{k=0}^{\infty} \mathrm{E}\left|\xi_{1}\right|^{q} I\left\{k \epsilon \leq\left|\xi_{1}\right|<(k+1) \epsilon\right\} \sum_{n=[k / 2]}^{\infty} n^{1-q} \\
& +C \epsilon^{2-q} \sum_{k=b(\epsilon)+1}^{\infty} k^{2-q} \mathrm{E}\left|\xi_{1}\right|^{q} I\left\{k \epsilon \leq\left|\xi_{1}\right|<(k+1) \epsilon\right\}
\end{aligned}
$$

$(3.22) \leq C \mathrm{E}\left|\xi_{1}\right|^{2}$.

Finally, by (3.20), we will get

$$
\begin{aligned}
M_{223}= & \sum_{n=b(\epsilon)+1}^{\infty} \int_{0}^{\infty} n^{-q}(x+\epsilon)^{1-q} \sum_{i=-\infty}^{\infty}\left|a_{n i}\right|^{q} \mathrm{E}\left|\xi_{1}\right|^{q} I\left\{n \epsilon<\left|a_{n i} \xi_{1}\right| \leq n(x+\epsilon)\right\} d x \\
\leq & C \sum_{n=b(\epsilon)+1}^{\infty} n^{-q} \mathrm{E}\left|\xi_{1}\right|^{q} I\left\{\left|\tilde{a} \xi_{1}\right|>n \epsilon\right\} \int_{0}^{\infty}(x+\epsilon)^{1-q} \sum_{i=-\infty}^{\infty}\left|a_{n i}\right|^{q} I\left\{\left|a_{n i} \xi_{1}\right| \leq n(x+\epsilon)\right\} d x \\
\leq & C \sum_{n=b(\epsilon)+1}^{\infty} n^{-q} \mathrm{E}\left|\xi_{1}\right|^{q} I\left\{\left|\xi_{1}\right|>n \epsilon\right\} \int_{0}^{\infty}(x+\epsilon)^{1-q} \sum_{j=1}^{\infty}\left(\sharp I_{n j}\right) j^{-q} \\
& \left(\sum_{k=0}^{2 n}+\sum_{k=2 n+1}^{(j+1) n}\right) I\left\{k(x+\epsilon) \leq\left|\xi_{1}\right|<(k+1)(x+\epsilon)\right\} d x \\
\leq & C \sum_{n=b(\epsilon)+1}^{\infty} n^{-1} \mathrm{E}\left|\xi_{1}\right|^{2} I\left\{\left|\xi_{1}\right|>n \epsilon\right\} \\
(3.23) \leq & C \mathrm{E}\left|\xi_{1}\right|^{2} I\left\{\left|\xi_{1}\right|>\epsilon^{-1}\right\}\left(\log ^{+}\left|\xi_{1}\right|-\log \epsilon\right) .
\end{aligned}
$$

Combining (3.19), (3.20), (3.21), (3.22), and (3.23), (3.18) is derived.

\section{Proof of Theorem 2.2}

Proof of Theorem 2.2. Since $\mathrm{E}\left|S_{n}\right|{ }^{p} I\left\{\left|S_{n}\right| \geq n \epsilon\right\}=\mathrm{P}\left(\left|S_{n}\right| \geq n \epsilon\right)$ when $p=0$, we only discuss the case of $0<p<2$. Note that

$$
\begin{aligned}
& \sum_{n=1}^{\infty} \frac{1}{n^{p}} \mathrm{E}\left|S_{n}\right|^{p} I\left\{\left|S_{n}\right| \geq n \epsilon\right\} \\
= & \epsilon^{p} \sum_{n=1}^{\infty} \mathrm{P}\left\{\left|S_{n}\right| \geq n \epsilon\right\}+\sum_{n=1}^{\infty} \frac{1}{n^{p}} \int_{n \epsilon}^{\infty} p x^{p-1} \mathrm{P}\left\{\left|S_{n}\right| \geq x\right\} d x .
\end{aligned}
$$


By (1.3), we only need to show that

$$
\lim _{\epsilon \searrow 0} \epsilon^{2-p} \sum_{n=1}^{\infty} \frac{1}{n^{p}} \int_{n \epsilon}^{\infty} p x^{p-1} \mathrm{P}\left\{\left|S_{n}\right| \geq x\right\} d x=\frac{p}{2-p}
$$

By Proposition 4.1 in [6], we have

$$
\lim _{\epsilon \searrow 0} \epsilon^{2-p} \sum_{n=1}^{\infty} \frac{1}{n^{p}} \int_{n \epsilon}^{\infty} p x^{p-1} \mathrm{P}(|N| \geq x / \sqrt{n}) d x=\frac{p}{2-p} .
$$

Hence we need show the following two propositions.

Proposition 4.1. One has

$$
\lim _{\epsilon \searrow 0} \epsilon^{2-p} \sum_{n=1}^{M b(\epsilon)} \frac{1}{n^{p}}\left|\int_{n \epsilon}^{\infty} p x^{p-1} P\left(\left|S_{n}\right| \geq x\right) d x-\int_{n \epsilon}^{\infty} p x^{p-1} P(|N| \geq x / \sqrt{n}) d x\right|=0 .
$$

Proof. It is easy to see

$$
\begin{aligned}
& \sum_{n=1}^{M b(\epsilon)} \frac{1}{n^{p}}\left|\int_{n \epsilon}^{\infty} p x^{p-1} \mathrm{P}\left(\left|S_{n}\right| \geq x\right) d x-\int_{n \epsilon}^{\infty} p x^{p-1} \mathrm{P}(|N| \geq x / \sqrt{n}) d x\right| \\
\leq & \sum_{n=1}^{M b(\epsilon)} \frac{1}{n^{p / 2}} n^{p / 2} \int_{0}^{\infty} \mathrm{P}(x+\epsilon)^{p-1}\left|\mathrm{P}\left(\left|S_{n}\right| \geq n(x+\epsilon)\right)-\mathrm{P}(|N| \geq \sqrt{n}(x+\epsilon))\right| d x \\
= & : \sum_{n=1}^{M b(\epsilon)} \frac{1}{n^{p / 2}}\left(\Delta_{n 1}^{\prime}+\Delta_{n 2}^{\prime}\right),
\end{aligned}
$$

where

$$
\begin{aligned}
\Delta_{n 1}^{\prime} & =n^{p / 2} \int_{0}^{1 / \sqrt{n} \Delta_{n}^{1 / 2 p}} \mathrm{P}(x+\epsilon)^{p-1}\left|\mathrm{P}\left(\left|S_{n}\right| \geq n(x+\epsilon)\right)-\mathrm{P}(|N| \geq \sqrt{n}(x+\epsilon))\right| d x \\
(4.4) & \leq \Delta_{n} n^{p / 2}\left(\frac{1}{\sqrt{n} \Delta_{n}^{1 / 2 p}}+\epsilon\right)^{p} \leq\left(\Delta_{n}^{1 / 2 p}+\sqrt{M} \Delta_{n}^{1 / p}\right)^{p},
\end{aligned}
$$

Since $n \leq M b(\epsilon)$ implies $\sqrt{n} \epsilon \leq \sqrt{M}$, by Markov's inequality,

$$
\begin{aligned}
\Delta_{n 2}^{\prime} & =n^{p / 2} \int_{1 / \sqrt{n} \Delta_{n}^{1 / 2 p}}^{\infty} \mathrm{P}(x+\epsilon)^{p-1}\left|P\left(\left|S_{n}\right| \geq n(x+\epsilon)\right)-\mathrm{P}(|N| \geq \sqrt{n}(x+\epsilon))\right| d x \\
(4.5) & \leq C n^{p / 2} \int_{1 / \sqrt{n} \Delta_{n}^{1 / 2 p}}^{\infty} \frac{1}{n(x+\epsilon)^{3-p}} d x \leq C \Delta_{n}^{1 / p-1 / 2} .
\end{aligned}
$$

Combining (4.4) and (4.5), (4.3) is derived.

Proposition 4.2. One has

$$
\lim _{M \rightarrow \infty} \limsup _{\epsilon \searrow 0} \epsilon^{2-p} \sum_{n=M b(\epsilon)+1}^{\infty} \frac{1}{n^{p}}\left|\int_{n \epsilon}^{\infty} p x^{p-1} P\left(\left|S_{n}\right| \geq x\right) d x-\int_{n \epsilon}^{\infty} p x^{p-1} P(|N| \geq x / \sqrt{n}) d x\right|=0 .
$$


Proof. Define $U_{n}^{\prime}$ as in Proposition 3.2. We have

$$
\begin{aligned}
& \sum_{n=M b(\epsilon)+1}^{\infty} \frac{1}{n^{p}}\left|\int_{n \epsilon}^{\infty} p x^{p-1} \mathrm{P}\left(\left|S_{n}\right| \geq x\right) d x-\int_{n \epsilon}^{\infty} p x^{p-1} \mathrm{P}(|N| \geq x / \sqrt{n}) d x\right| \\
\leq & \sum_{n=M b(\epsilon)+1}^{\infty} \int_{0}^{\infty} p(x+\epsilon)^{p-1} \mathrm{P}(|N| \geq \sqrt{n}(x+\epsilon)) d x \\
& +\sum_{n=M b(\epsilon)+1}^{\infty} \int_{0}^{\infty} p(x+\epsilon)^{p-1} \mathrm{P}\left(\left|S_{n}\right| \geq n(x+\epsilon)\right) d x \\
\leq & K_{1}+K_{2} .
\end{aligned}
$$

For $K_{1}$, by the Markov inequality,

$$
\begin{aligned}
K_{1} & \leq C \sum_{n=M b(\epsilon)+1}^{\infty} \int_{0}^{\infty} p(x+\epsilon)^{p-1} \frac{\mathrm{E}|N|^{4}}{n^{2}(x+\epsilon)^{4}} d x \\
& \leq C \sum_{n=M b(\epsilon)+1}^{\infty} n^{-2} \epsilon^{p-4} \\
& \leq C M^{-1} \epsilon^{p-2} .
\end{aligned}
$$

Therefore,

$$
\lim _{M \rightarrow \infty} \limsup _{\epsilon \searrow 0} \epsilon^{2-p} K_{1}=0
$$

It is easy to see

$$
\begin{aligned}
K_{2} \leq & \sum_{n=M b(\epsilon)+1}^{\infty} \int_{0}^{\infty} p(x+\epsilon)^{p-1}\left[\mathrm{P}\left(\sup _{i}\left|a_{n i} \xi_{i}\right|>n(x+\epsilon)\right)\right. \\
& \left.+\mathrm{P}\left(\left|U_{n}^{\prime}-E U_{n}^{\prime}\right| \geq n(x+\epsilon) / 2\right)\right] d x \\
= & : K_{21}+K_{22} .
\end{aligned}
$$

Similar with the proof of (3.10), we can get

$$
\begin{aligned}
K_{21} & \leq C \mathrm{E}\left|\xi_{1}\right| \int_{0}^{\infty} \sum_{n=M b(\epsilon)+1}^{\infty}(x+\epsilon)^{p-2} \sum_{k=n}^{\infty} I\left\{k(x+\epsilon) \leq\left|\xi_{1}\right|<(k+1)(x+\epsilon)\right\} d x \\
& \leq C \mathrm{E} \xi_{1}^{2} \int_{0}^{\infty}(x+\epsilon)^{p-3} \sum_{k=M b(\epsilon)+1}^{\infty} I\left\{k(x+\epsilon) \leq\left|\xi_{1}\right|<(k+1)(x+\epsilon)\right\} d x \\
& \leq C \mathrm{E}\left|\xi_{1}\right|^{2} I\left\{\left|\xi_{1}\right| \geq M \epsilon^{-1}\right\} \int_{0}^{\infty}(x+\epsilon)^{p-3} d x \\
& \leq C \epsilon^{p-2} \mathrm{E}\left|\xi_{1}\right|^{2} I\left\{\left|\xi_{1}\right| \geq M \epsilon^{-1}\right\} .
\end{aligned}
$$


So we have

$$
\lim _{M \rightarrow \infty} \limsup _{\epsilon \searrow 0} \epsilon^{2-p} K_{21}=0 .
$$

Note that $\sum_{m=1}^{\infty} \phi^{1 / 2}(m)<\infty$, by Lemma 2.2 , for $q>2$,

$$
\begin{aligned}
K_{22} \leq & \sum_{n=M b(\epsilon)+1}^{\infty} \int_{0}^{\infty} p n^{-q}(x+\epsilon)^{p-1-q}\left\{\left(\sum_{i=-\infty}^{\infty} \mathrm{E}\left(a_{n i} \xi_{1}\right)^{2} I\left\{\left|a_{n i} \xi_{1}\right| \leq n(x+\epsilon)\right\}\right)^{q / 2}\right. \\
& +\sum_{i=-\infty}^{\infty} \mathrm{E}\left|a_{n i} \xi_{1}\right|^{q} I\left\{\left|a_{n i} \xi_{1}\right| \leq n \epsilon\right\} \\
& \left.+\sum_{i=-\infty}^{\infty} \mathrm{E}\left|a_{n i} \xi_{1}\right|^{q} I\left\{n \epsilon<\left|a_{n i} \xi_{1}\right| \leq n(x+\epsilon)\right\}\right\} d x \\
=: & K_{221}+K_{222}+K_{223} .
\end{aligned}
$$

Here we omit the proof of $K_{221}$ because it is routine. Next we will estimate $K_{222}, K_{223}$.

$$
\begin{aligned}
K_{222} \leq & \epsilon^{p-q} \sum_{n=M b(\epsilon)+1}^{\infty} n^{-q} \sum_{i=-\infty}^{\infty}\left|a_{n i}\right|^{q} \mathrm{E}\left|\xi_{1}\right|{ }^{q} I\left\{\left|a_{n i} \xi_{1}\right| \leq n \epsilon\right\} \\
\leq & \epsilon^{p-q} \sum_{n=M b(\epsilon)+1}^{\infty} n^{-q} \sum_{j=1}^{\infty}\left(\sharp I_{n j}\right) j^{-q} \sum_{k=0}^{(j+1) n} \mathrm{E}\left|\xi_{1}\right|^{q} I\left\{k \epsilon \leq\left|\xi_{1}\right|<(k+1) \epsilon\right\} \\
\leq & \epsilon^{p-q} \sum_{n=M b(\epsilon)+1}^{\infty} n^{-q} \sum_{j=1}^{\infty}\left(\sharp I_{n j}\right) j^{-q}\left\{\sum_{k=0}^{2 n}+\sum_{k=2 n+1}^{(j+1) n}\right\} \mathrm{E}\left|\xi_{1}\right|^{q} I\left\{k \epsilon \leq\left|\xi_{1}\right|<(k+1) \epsilon\right\} \\
\leq & C \epsilon^{p-q} \sum_{n=M b(\epsilon)+1}^{\infty} n^{1-q} \sum_{k=0}^{2 n} \mathrm{E}\left|\xi_{1}\right|^{q} I\left\{k \epsilon \leq\left|\xi_{1}\right|<(k+1) \epsilon\right\} \\
& +C \epsilon^{p-q} \sum_{n=M b(\epsilon)+1}^{\infty} n^{-q} \sum_{k=2 n+1}^{\infty} n\left(\frac{k}{n}\right)^{-(q-1)} \mathrm{E}\left|\xi_{1}\right|^{q} I\left\{k \epsilon \leq\left|\xi_{1}\right|<(k+1) \epsilon\right\} \\
\leq & C \epsilon^{p-q} \sum_{k=0}^{\infty} \mathrm{E}\left|\xi_{1}\right|^{q} I\left\{k \epsilon \leq\left|\xi_{1}\right|<(k+1) \epsilon\right\} \sum_{n=[k / 2]}^{\infty} n^{1-q} \\
& +C \epsilon^{p-q} \sum_{k=M b(\epsilon)+1}^{\infty} k^{2-q} \mathrm{E}\left|\xi_{1}\right|^{q} I\left\{k \epsilon \leq\left|\xi_{1}\right|<(k+1) \epsilon\right\} \\
(4.10) \leq & C \epsilon^{p-2} \mathrm{E}\left|\xi_{1}\right|^{2} I\left\{\left|\xi_{1}\right|>M \epsilon^{-1}\right\} .
\end{aligned}
$$

Finally, we will get

$$
K_{223}=\sum_{n=M b(\epsilon)+1}^{\infty} \int_{0}^{\infty} p n^{-q}(x+\epsilon)^{p-1-q} \sum_{i=-\infty}^{\infty} \mathrm{E}\left|a_{n i} \xi_{1}\right|^{q} I\left\{n \epsilon<\left|a_{n i} \xi_{1}\right| \leq n(x+\epsilon)\right\} d x
$$




$$
\begin{aligned}
\leq & C \sum_{n=M b(\epsilon)+1}^{\infty} n^{-q} \mathrm{E}\left|\xi_{1}\right|^{q} I\left\{\left|\tilde{a} \xi_{1}\right|>n \epsilon\right\} \\
& \int_{0}^{\infty}(x+\epsilon)^{p-1-q} \sum_{j=1}^{\infty}\left(\sharp I_{n j}\right) j^{-q} \sum_{k=0}^{(j+1) n} I\left\{k(x+\epsilon) \leq\left|\xi_{1}\right|<n(x+\epsilon)\right\} d x \\
\leq & C \sum_{n=M b(\epsilon)+1}^{\infty} n^{-q} \mathrm{E}\left|\xi_{1}\right|{ }^{q} I\left\{\left|\xi_{1}\right|>n \epsilon\right\} \int_{0}^{\infty}(x+\epsilon)^{p-1-q} \sum_{j=1}^{\infty}\left(\sharp I_{n j}\right) j^{-q} \\
& \left(\sum_{k=0}^{2 n}+\sum_{k=2 n+1}^{(j+1) n}\right) I\left\{k(x+\epsilon) \leq\left|\xi_{1}\right|<(k+1)(x+\epsilon)\right\} d x \\
\leq & C \sum_{n=M b(\epsilon)+1}^{\infty} n^{1-p} \mathrm{E}\left|\xi_{1}\right|^{p} I\left\{\left|\xi_{1}\right|>n \epsilon\right\} \\
(4.11) \leq & \epsilon^{p-2} \mathrm{E}\left|\xi_{1}\right|^{2} I\left\{\left|\xi_{1}\right|>M \epsilon^{-1}\right\} .
\end{aligned}
$$

Then we obtain

$$
\lim _{M \rightarrow \infty} \limsup _{\epsilon \searrow 0} \epsilon^{2-p} K_{22}=0 .
$$

Combining (4.8), (4.9), and (4.12), then (4.6) is derived.

\section{References}

[1] P. Billingsley, Convergence of Probability Measures, Wiley, New York, 1968.

[2] R. M. Burton and H. Dehling, Large deviations for some weakly dependent random process, Statist. Probab. Lett. 9 (1990), no. 5, 397-401.

[3] P. L. Hsu and H. Robbins, Complete convergence and the strong law of large numbers, Proc. Nat. Acad. Sci. U.S.A. 33 (1947), 25-31.

[4] D. L. Li, M. B. Rao, and X. C. Wang, Complete convergence of moving average processes, Statist. Probab. Lett. 14 (1992), no. 2, 111-114.

[5] Y. X. Li, Precise asymptotics in the law of large numbers of moving-average processes, Acta Math. Sci. Ser. A Chin. Ed. 26 (2006), no. 5, 675-687.

[6] W. D. Liu and Z. Y. Lin, Precise asymptotics for a new kind of complete moment convergence, Statist. Probab. Lett. 76 (2006), no. 16, 1787-1799.

[7] Q. M. Shao, A moment inequality and its application, Acta Math. Sinica 31 (1988), no. $6,736-747$.

[8] X. Y. Yang, The law of the iterated logarithm and the central limit theorem with random indices for B-valued stationary linear processes, Chinese Ann. Math. Ser. A 17 (1996), no. $6,703-714$.

[9] L. X. Zhang, Complete convergence of moving average processes under dependence assumptions, Statist. Probab. Lett. 30 (1996), no. 2, 165-170.

School of Mathematics and Statistics

Zhejiang University of FinANCe and Economics

Hangzhou 310018, P. R. China

E-mail address: lijiezufe@gmail.com 